ponsible for the bizarre postures could not be determined during subsequent examinations. One group 2 patient showed a state exactly resembling transient global amnesia. Neurological abnormalities consistently improved in cases who survived.

In this series the incidence of intellectual impairment was high (17\% of all patients, $24 \%$ of survivors). Previous studies suggest that neurological outcome after cardiac arrest is good. These studies were retrospective however, or emphasized satisfactory cardiovascular outcome without careful neurological follow up or considered only patients managed in coronary care areas (where immediate resuscitation is the rule). None of our cases surviving with intellectual impairment suffered their initial cardiac arrest in such an area.

Disparaging claims have been made about the use of clinical assessments in determining outcome after cardiac arrest (Bokonjic and Buchthal, 1961; Pampiglione and Harden, 1968; Plum and Posner, 1972), probably through inadequate documentation of sufficient cases. From our cases it is evident that a patient showing group 1 characteristics at any time more than one hour after cardiac arrest will have a poor prognosis. Two group 1 patients recovered consciousness by four hours, however, and were then not clearly separable from those who survived normally. Therefore an examination at one hour, yields more discriminatory findings.

The nature of the anoxic injury which results in the shortterm disturbances described (table III) is probably a nonstructural intraneuronal (metabolic) defect. The processes which have been found to be disturbed depend on the integrity of large scattered functional groups of cells, and being so they are especially susceptible to environmental disturbances of any sort. The return of these functions over hours or days can be accounted for by a period of intraneuronal repair after withdrawal of the noxious stimulus, similar to other metabolic encephalopathies. Some focal reversible disturbances were seen, but focal features have been described in patients with metabolic encephalopathy (Meyer and Portnoy, 1958; Pearce, 1963; Locke et al., 1961). A vascular-occlusive aetiology of the reversible disturbances is unlikely because most of the disturbances could not be accounted for by a focal lesion, and the rapid resolution of the disturbances contrasts with the delayed or incomplete recovery seen with cerebral infarctions. Cerebral oedema does not account for the transient disturbances either because in this situation it is rare (Brierley, 1961), and it usually manifests itself some time after the arrest, whereas the transient disturbances were present immediately.

Of interest is the absence of some findings which might have been expected. Instances of focal cerebral infarction as commonly occur in patients with vascular disease were not seen in our patients nor did patients show an association of features (pupillary abnormalities, oculomotor signs, papilloedema) suggestive of cerebral oedema. Though the cerebellum is known to be sensitive to anoxia, evidence of cerebellar damage was not present in any of our patients who could adequately be assessed. The rare occurrence of delayed neurological deterioration which has been described by other workers (Plum et al., 1962) was not seen in our cases.

A larger prospective series would almost certainly show that other reversible neurological abnormalities occasionally occur after cardiac arrest and might also serve to establish the optimum time and exact reliability for this clinical method of determining outcome.

The authors wish to thank Dr. R. J. Burns and Dr. A. R. Clarkson, who commented in depth on every aspeot of the text; Dr. J. V. Gordon, Dr. R. J. Burns, and Dr. J. P. Rice, who advised on the protocol for clinical assessment; and the medical staff of the Royal Adelaide Hospital, who gave permission for their cases to be examined.

\section{References}

Binnie, C. D., et al. (1970). British Medical fournal, 4, 265.

Bokonjic, N., and Buchthal, F. (1961). In Cerebral Anoxia and the Electroencephalogram, ed. J. S. Meyer and H. Gastaut, p. 118. Springfield,

Brierley, J. B., et al. (1971). Lancet, 2, 560.

Brierley, J. B. (1961). In Cerebral Anoxia and the Electroencephalogram, ed. J. S. Meyer and H. Gastaut, p. 167. Springfield, Thomas.

Dobson, M., et al. (1971). British Medical Fournal, 3, 207.

Hockaday, J. M., et al. (1965). Electroencephalography and Clinical Neurophysiology, 18, 575 .

Johnson, L. A., et al. (1967). American fournal of Cardiology, 20, 831.

Lancet, (1973). 1, 523. Lancet, (1973). 1, 5errill, J. P., and Tyler, H. R. (1961). Archives of Internal
Medicine, 108, 519.

McNamee, B. T., et al. (1970). British Medical fournal, 4, 204.

Meyer, J. S., and Portnoy, H. D. (1958). Neurology, 8, 601

Pampiglione, G., and Harden, A. (1968). Lancet, 1, 1261.

Pearce, J. M. S.' (1963). Postgraduate Medical fournal, 39, 653.

Plum, F., Posner, J. B., and Hain, R. F. (1962). Archives of Internal Medicine,

110, 18.
Plum, F., and Posner, J. B. (1972). The Diagnosis of Stupor and Coma. Philadelphia, Davis.

\title{
Behaviour of Standard Gravity-fed Administration Sets Used for Intravenous Infusion
}

\section{Introduction}

Intravenous infusion is widely used in hospitals for the administration of bulk fluids, including blood, drugs, and food in liquid form. The standard infusion equipment consists of a bottle or bag containing the fluid to be administered, a plastic administration set, and a suitable cannula for insertion into the vein. Administration sets are manufactured in two basic forms, one for adult use and the other for paediatric use. About 3 million adult sets are used in the U.K. every year.

The standard administration set is gravity-fed, the rate of flow being controlled by a simple plastic regulating clamp. Two alternative methods for the administration of fluids are the pressure pump and the peristaltic pump. The former generally employs a syringe principle, the plunger being driven at a speed selected so that a given volume of fluid is delivered in a 
set time. The peristaltic pump delivers small equal quantities of fluid by periodically squeezing the delivery tube of the giving set using a series of occluding fingers. A bolus of liquid is thus driven along the tube. Both methods depend on relatively expensive equipment and hence are not widely used in general wards, where the standard gravity-fed administration set reigns supreme.

It is generally recognized by the hospital staff who set up and use the standard administration set of whichever brand that it has a number of deficiencies. It is the purpose of this paper to describe the behaviour of typical sets in controlled laboratory experiments and in hospital use. The causes of the variation of infusion rate with time are examined and it is shown that for accurate infusion some type of automatic regulator must be used.

\section{Construction and Mode of Action}

The construction of a typical adult administration set is shown in fig. 1. The fluid to be administered is contained in a bottle or bag (B). The pressure above the liquid is kept at or near atmospheric pressure. When the administration set is connected to $B$ fluid enters the filter chamber $(F)$, which is usually maintained full. The fluid passes through a small plastic tube and drips into the drip chamber (D), which is usually maintained about onethird full. Fluid leaves $D$, passes down a long plastic tube (T), and enters the cannula $(C)$ inserted into a vein. $T$ is partially occluded by a plastic regulating clamp $(R)$ controlling the rate of flow into the patient. $B$ is usually held in a stand so that the fluid level in $B$ is about $1 \mathrm{~m}$ above the cannula.

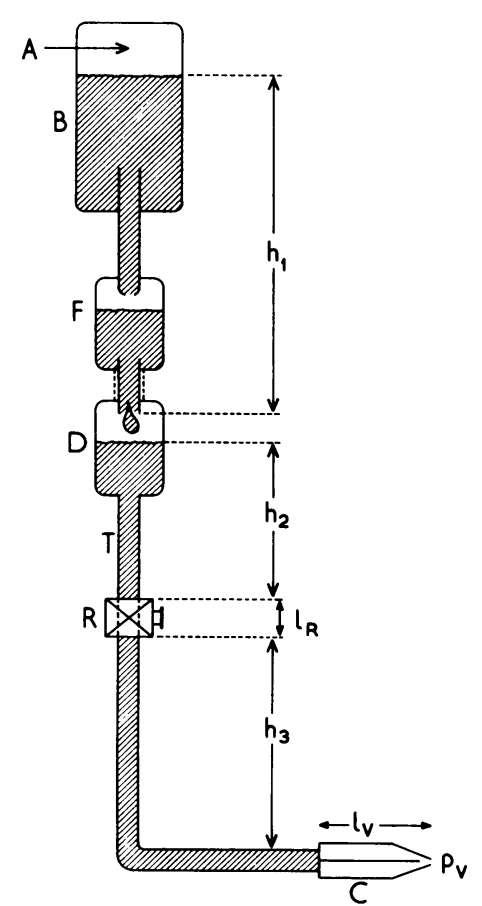

FIG. 1 - Construction of typical adult administration set. $A=$ Atmospheric pressure above liquid. $B=$ Bottle or bag. $F=$ Filter chamber. $D=$ Drip chamber. $T=$ Plastic tube. $\mathbf{R}=$ Regulating clamp. $C=$ Cannula. $1_{R}$ is effective length of occluded piece of $T$, and $1 v$ is length of cannula. $P_{Y}$ is absolute venous pressure at cannula. Lengths $h_{1} h_{2}$ and cannula. py is heights of columns of liquid in various sections.

All administration sets used in the U.K. must meet B.S. specification 2463:1962.* According to statements made on the containers of commonly used adult administration sets the rate of infusion may be calculated from the fact that 15 drops, counted in the drip chamber, is approximately equal to $1 \mathrm{ml}$ of fluid. Two problems immediately arise. Firstly, if, for example, 11. of fluid is to be administered in eight hours a ready reckoner is required to convert the information into drops/min, which is the quantity that the nurse sets using the regulating clamp. Rarely is such an aid available. Secondly, it is well known that the drip rate affects the size of the drops, and several workers have described this phenomenon (La Cour, 1965, 1966; Ferenchak et al., 1971). In general the lower the drip rate the smaller the drop formed, so that the number of drops per $\mathrm{ml}$ rises. We have studied the variation of drop size with drip rate of saline and dextrose for a number of blood administration sets manufactured by Baxter Division, Travenol Laboratories Ltd., and Avon Medicals Ltd., and have found over the range of rates commonly used that the numbers of drops per $\mathrm{ml}$ vary from 16 to over 18, the larger drops corresponding to the higher rates. The nature of the fluid to be administered will also affect the size of the drop formed. This is due to the surface tension of the fluid; since this tension is temperature dependent drop size is affected by temperature, though the effect is small.

The regulating clamp used to control the drop rate usually consists of a wheel which can roll along an inclined plane. The plastic tube $T$ (fig 1) is trapped between the wheel and the plane. Rolling the wheel up the plane increasingly occludes $T$. This device is made entirely of plastic and experience shows that control of drip rate using it is difficult. Apart from occasional mechanical failures such as the wheel popping out of its guides the assembly is easy to deform. Thus a set rate may change immediately the hand leaves the regulating clamp. The device does not allow a fine enough control over the drip rate, so that it is difficult to set a rate to within \pm 2 drops $/ \mathrm{min}$.

These points indicate the difficulties encountered in calculating a required drip rate and in trying to set up a drip rate using the usual regulating clamp. Some administration sets have been supplied with a plastic screw device to control the drip rate but most of the criticisms still apply. It should also be noted that a nurse who times drops for, say, 15 seconds may introduce a large error into the total infusion time. For a required drip rate of $40 / \mathrm{min}$, if the number timed is only 8 or 9 per 15 seconds then an error of $10-20 \%$ arises and the total infusion time will be increased by the same percentage.

\section{Present Investigation}

Once a given drip rate has been established it is commonly observed that this rate may be subject to extremely wide variations and thus the chances of a patient receiving an infusion in a controlled manner over the correct period are remote. When it is necessary for the patient to have a well controlled infusion, such as in the administration of oxytocin, constant supervision of the rate is essential though wasteful and expensive of staff time.

To determine the factors causing these variations two separate studies of administration sets were carried out. The first was a laboratory study in which factors such as pressure could be controlled accurately, and the second was a study of these rate variations in a number of administration sets being used routinely in large general wards.

\section{LABORATORY STUDY}

Administration sets marketed by different manufacturers were each fed with water from a constant pressure head device (equivalent to maintaining a constant level in bag B). Cannulae of various sizes normally used for intravenous infusion were attached to the distal ends of the sets. An initial drip rate was set using the regulator clamp and the drip rate was measured as a function of time over several hours. The water issuing from 
the cannula was collected in a beaker and atmospheric pressure was maintained at the cannula exit. All sets showed similar variations of drip rate with time (fig. 2).

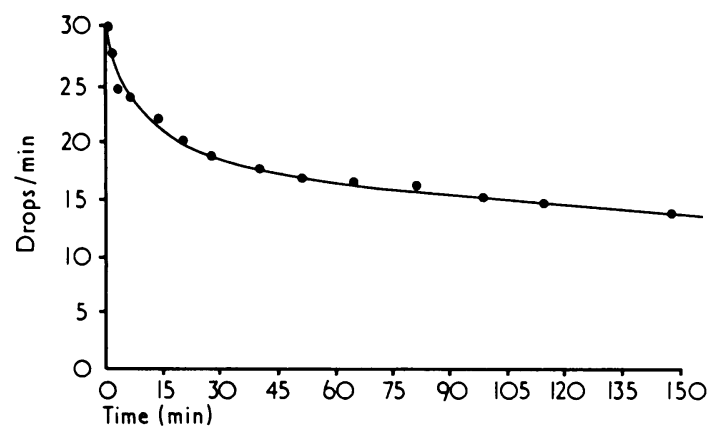

FIG. 2-Variation in drip rate with time for administration set adjusted initially to $30 \mathrm{drops} / \mathrm{min}$. Pressure head was maintained constant and exit pressure from cannula was atmospheric.

From an initial rate set at just over 30 drops/min the rate fell to just over 16 drops/min in about 60 minutes. The fall continued, though more slowly, until at the end of four hours the rate was under $15 \mathrm{drops} / \mathrm{min}$. The variation of rate with time is of exponential form, and a semi-log plot of drip rate versus time is shown in fig. 3. Two linear regions are evident, indicating that an initial process with a short time constant gives way to a second process with a much longer time constant. These results are indicative of creep in the material of either the plastic tube or the regulating clamp or both. The first region with a short time constant corresponds to primary creep and the second region to secondary creep. This behaviour was evident in all the sets.

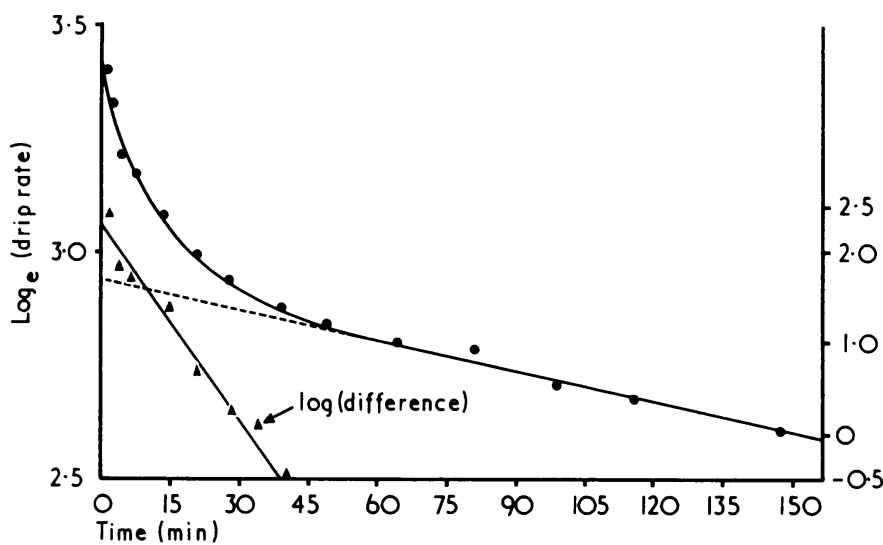

FIG. 3-Data from fig. 2 plotted on logarithmic scale showing existence of two time constants. Curve labelled log (difference) was obtained by extrapolating back via dashed line so as to be able to subtract effect of long time constant at early times.

To establish the source of creep a metal regulating clamp was used on some sets. Similar results were obtained, and since with the forces involved the creep of the metal was negligible it was ascertained that the major source of creep causing the drip rate variation lay in the plastic material of the tube. A number of different designs of metal regulating clamp were tried and though some were much easier to use than the standard clamp the variation of drip rate with time was essentially unaltered.

Both primary and secondary creep are temperature dependent and a series of experiments were conducted using a metal regulating clamp held at temperatures from $0^{\circ}$ to $50^{\circ} \mathrm{C}$. The variation in drip rate was dependent on the temperature of the regulating clamp and hence the temperature of the plastic tube trapped by the clamp (fig. 4). Increases of temperature decreased the total change in drip rate over the period of the experiment. These results are in accordance with the known variation of creep with temperature (Williams, 1973), but from the point of view of infusion of given volumes of fluid in set times conditions are far from ideal even using raised tube temperature in the region of the regulating clamp.

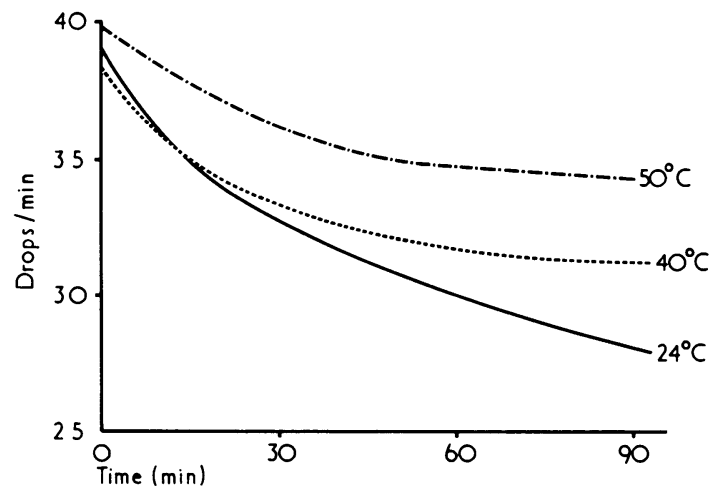

FIG. 4-Variation of drip rate with time with metal regulating clamp maintained at different temperatures.

Several subsidiary experiments were carried out on the administration sets. An estimate of the stress required to occlude the infusion tube completely was made by compressing it with a rectangular bar of square cross-section, side $0.6 \mathrm{~cm}$. The area compressed was $0.48 \mathrm{~cm}^{2}$ and the force required to stop the flow $55.6 \mathrm{~N}(5.7 \mathrm{kgf})$. The mean stress was thus $11.6 \times$ $10^{5} \mathrm{~N} / \mathrm{m}^{2}\left(11.8 \mathrm{kgf} / \mathrm{cm}^{2}\right)$. An estimate of the area compressed by the usual plastic regulating clamp is $0.4 \mathrm{~cm}^{2}$ and the stress must therefore be of the order of $10^{6} \mathrm{~N} / \mathrm{m}^{2}$, at which level creep effects are likely to be exhibited.

A study was also made of the bore of the infusion tube when almost fully occluded. A micrometer screw gauge was used to pinch the tube and measure the change in dimension of the tube from just zero drip rate to a rate of about $30 \mathrm{drops} / \mathrm{min}$. The change was found to be approximately $0.0076 \mathrm{~cm}$. The crosssection of the tube at the clamping point during control by the regulating clamp was examined and the tube configuration for drip rates commonly used is shown in fig. 5 . The centre portion is completely occluded; the large stresses required for reducing the drip rate to zero are employed to occlude the two small bores indicated in the diagram. For accurate setting of the drip rate it is thus necessary to use a regulating clamp having a large movement of the wheel corresponding to a small change in tube dimension. The usual regulating clamps supplied with administration sets do not meet this specification.

Finally, the pressure drop across the cannula was measured using simple manometer techniques. Simultaneous measurement of the volume flow rate showed that it was proportional to the pressure difference across the cannula, in agreement with the Poiseuille equation for the slow flow of a viscous fluid. The pressure at the exit of the cannula was varied from atmospheric to $50 \mathrm{~cm} \mathrm{H}_{2} \mathrm{O}$ above atmospheric and similar results were obtained for drip rates up to approximately $100 / \mathrm{min}$.

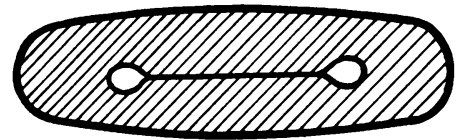

FIG. 5-Cross-section of tube at clamping point during control by regulating clamp. 


\section{CLINICAL STUDY}

By far the most common and certainly the most loudly voiced complaint by users of standard administration sets is that once a drip rate has been set it is subject to wide variations. For such a common complaint it is interesting to note that very few hospital staff have any idea of the magnitude of the variations and there appears to be little if any published work on this topic. Measurements were therefore carried out to determine the variation in drip rate under general ward conditions.

A small electric light bulb and photosensitive device were combined to form a "drip detector" which could be clamped around the drip chamber of any standard administration set. When a drip fell through the chamber the output of the photosensitive device changed and the signal, after amplification, was used to drive the pen of a chart recorder. Thus each time a drop passed the sensing head a mark was made on the recorder paper. The paper was run through the recorder at a constant speed and thus the number of drips per minute could be counted at any particular time during the infusion.

The measurements were made for a number of patients receiving intravenous infusions of commonly used fluids. Though no two results were the same all showed wide variations from the set rate, and a typical (so far as such a word applies) plot of drip rate with time is shown in fig. 6 . The rate, initially set at $35 \mathrm{drops} / \mathrm{min}$, varied from zero to over $60 \mathrm{drops} / \mathrm{min}$ in the period of measurement. The variation in some patients was smaller but still significant and in others greater.

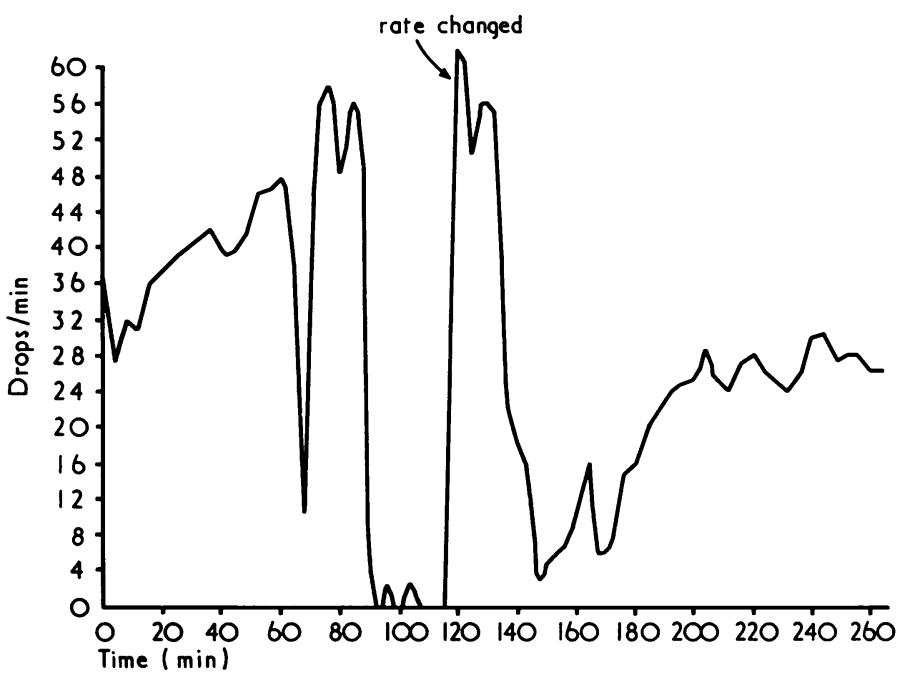

FIG. 6-Drip rate, set initially at 35 drops/min, as function of time for typical intravenous infusion given in genera ward. Compare with fig. 2.

When fig. 6 is compared with fig. 2, which shows how drip rate varies with time for administration sets run under basically constant pressure conditions, one fundamental point stands out. The variations shown in fig. 6 are caused by an effect which has nothing to do with the type of administration set used, the material of the set, or the type of regulating clamp used as there is no evidence of exponential (creep) decay, and no evidence of the decay was found in any of the measurements. The administration set cannot therefore be the cause of the variations. These can arise only with changes in pressure at the distal end of the cannula. That is to say, the variation in drip rate is caused by changes in the peripheral venous pressure of the patient, and this effect almost totally swamps all other effects.

To verify this conclusion simultaneous measurements of drip rate and venous pressure were made. The pressure transducer of a General Electric venous pressure monitor was connected to a cannula inserted into the vein and the output from the transducer displayed on a meter and chart recorder. The variations in drip rate were measured as described above. In order to measure the venous pressure at the exit of the cannula used for the intravenous infusion a vein with a $Y$ branch in the back of the hand was chosen. The infusion cannula was inserted into one arm of the $\mathrm{Y}$ and pushed past the junction into the main branch of the vein. The cannula with the pressure transducer attached was inserted into the other arm of the $Y$ and pushed along the vein until its end was level with that of the infusion cannula. At the end of the experiment each cannula was filled with radio-opaque solution. An $x$-ray film verified that the ends of the cannulae were close to one another.

Variations in drip rate with venous pressure over a period of time are shown in fig. 7. The pressure was varied by allowing the subject either to lie down or sit up in various positions. As he was allowed to see the pressure reading he could, by slightly altering position, keep the venous pressure reasonably constant over a period of time. In the initial part of the experiment where the subject was lying prone with a steady venous pressure a slight fall in drip rate with time is apparent, probably due to creep effects in the infusion tube. The tube had been partially occluded by the regulating clamp for some time previously and hence some adjustment of the creep effects had already occurred. Increasing the venous pressure by making the subject sit half up noticeably decreased the drip rate, and a further increase in venous pressure due to the subject sitting straight up caused yet another decrease in drip rate. In general, provided the venous pressure remained constant the drip rate remained constant.

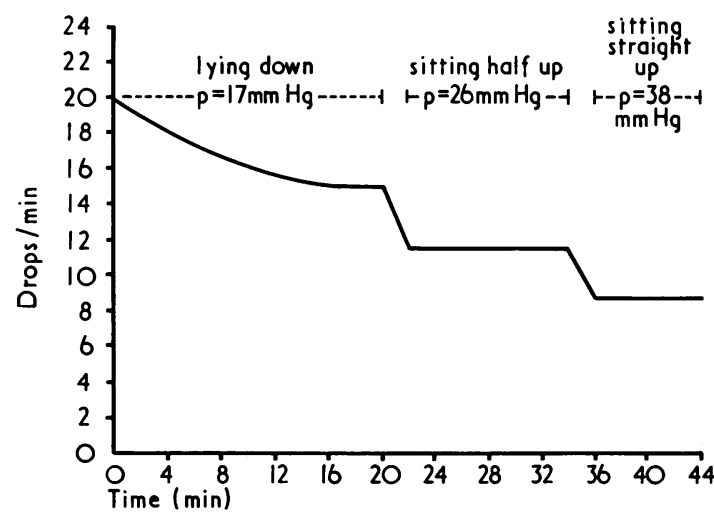

FIG. 7-Drip rate as function of time for subject changing position and keeping recorded venous pressure (p) steady at values indicated.

The variation of drip rate with venous pressure over a wide range of pressures is shown in fig. 8 to be reasonably linear. In particular, the drip rate becomes zero at a venous pressure of approximately $80 \mathrm{~mm} \mathrm{Hg}$ (almost $109 \mathrm{~cm} \mathrm{H}_{2} \mathrm{O}$ ). This is expected to occur when the total pressure head and the total venous pressure are equal. The measured height of the total pressure head was about $103 \mathrm{~cm}$, a value within experimental error of the figure read from fig. 8.

This evidence clearly shows that the drip rate is largely controlled by the venous pressure. Patients receiving intravenous infusions sit up, lie down, roll over, cough, etc., and all these movements affect venous pressure. Even when asleep changes in breathing rate and depth can alter the venous pressure. Thus variations in drip rate must be expected unless the patient's venous pressure remains constant.

\section{FACTORS CONTROLLING DRIP RATE}

The standard administration set is gravity-fed, the fluid flowing 


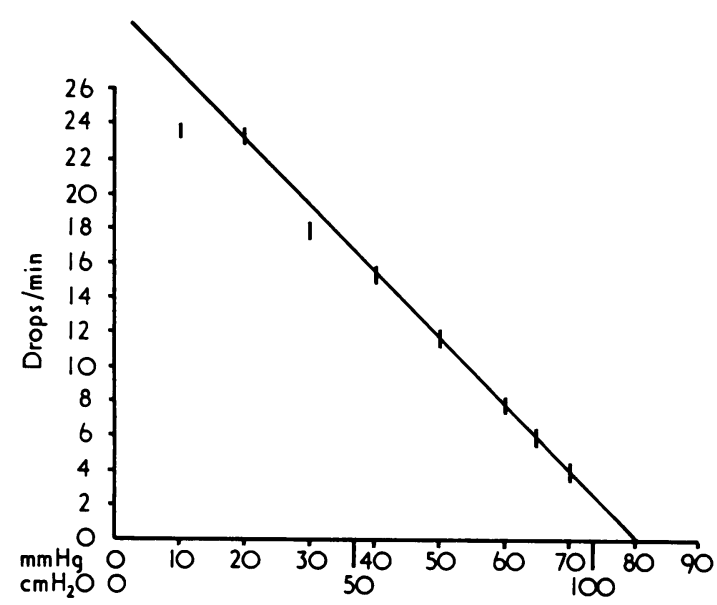

FIG. 8-Drip rate as function of venous pressure for same subject as in fig. 7 .

into the vein at a rate depending on hydrostatic pressures. The maximum value of the driving pressure is obtained by subtracting the venous pressure referred to atmosphere from the pressure due to the total height of fluid above the venous cannula. When the regulating clamp is controlling the flow an appreciable pressure drop occurs across the deformed region of the tube. The available driving pressure is therefore reduced from the maximum value. The cannula further reduces the pressure, affecting the flow in a way which depends on its length and diameter.

To examine these effects in detail consider the simplified diagram of an administration set shown in fig. 1 . The set is assumed to be vertical over the greater part of its length and horizontal for a short section near the cannula. The heights of the liquid in various parts of the set are indicated. Absolute venous pressure is given by the equation $\mathrm{p}_{\mathrm{v}}=\mathrm{A}{ }^{+\mathrm{p}}$ hydraulic ${ }^{-p}$ droplet ${ }^{-p}$ regulator ${ }^{-p}$ cannula. In this equation $A$ is the atmospheric pressure, approximately $1,000 \mathrm{~cm} \mathrm{H}_{2} \mathrm{O}$. Phydraulic is the sum of the hydrostatic pressures due to the heights of liquid $h_{1}, h_{3}$, and $h_{3}$. These total about $100 \mathrm{~cm} \mathrm{H}_{2} \mathrm{O}$. pdroplet represents the pressure change across the pendant drop and its value is less than $2 \mathrm{~cm} \mathrm{H}_{2} \mathrm{O}$. The terms ${ }^{\mathrm{p}}$ regulator and pcannula depend on the volume flow rate. In each case the pressure drop is directly proportional to this flow rate and may be calculated assuming Poiseuille flow. A typical value for p cannula is 2 or $3 \mathrm{~cm} \mathrm{H}_{2} \mathrm{O}$ when the flow rate corresponds to $30 \mathrm{drops} / \mathrm{min}$. If the absolute venous pressure $\mathrm{p}_{\mathrm{v}}$ in a vein in the forearm of a supine patient is assumed to be $10 \mathrm{~cm} \mathrm{H}_{2} \mathrm{O}$ above $\mathrm{A}$ the above equation becomes $10+\mathrm{A}=\mathrm{A}+100-2$ $-{ }^{\mathrm{p}}$ regulator - 3-that is, ${ }^{\mathrm{p}}$ regulator $=85 \mathrm{~cm} \mathrm{H}_{2} \mathrm{O}$. With this value it is possible to calculate an effective diameter for the constriction provided by the clamp. The calculated diameter is about $160 \mu \mathrm{m}$, through which the fluid (water, for example) flows at about $1.5 \mathrm{~m} / \mathrm{sec}$. Measurements on the pear-shaped bores shown in fig. 5 confirm that this diameter is a good estimate.

\section{Conclusions}

Laboratory and clinical trials have shown that the major factor influencing drip rate during intravenous infusion is the variation in the patient's venous pressure. Experiments have also shown that creep is present in the plastic tubing of the administration set but the change in drip rate due to this factor is completely overshadowed by venous pressure effects in the clinical situation. Controlled clinical experiments have shown that if the venous pressure remains constant the drip rate remains essentially constant, but this situation does not, of course, normally exist.

It therefore follows that the chance of administering a fluid at a uniform rate over a given time using the standard administration set is extremely low. In order to achieve a constant administration rate with the standard gravity-fed set it is necessary to use some form of servo-controlled regulating clamp. Then changes in venous pressures together with the other factors mentioned can be compensated by automatically altering the pressure drop across the regulating clamp, usually by varying the diameter of the infusion tube at this point in a controlled way.

We wish to express our sincere thanks to Professor D. Mattingly for early discussions on this problem and to thet medical and nursing staff of the Royal Devon and Exeter Hospital for their help with the clinical investigation. We are also indebted to Mr. H. A. J. Chidley and Mr. F. Miners, of the department of physics, University of Exeter, for designing and building much of the equipment used in these studies.

\section{References}

Ferenchak, P., Collins, J. J., and Morgan, M. D. (1971). Surgery, 70, 674. La Cour, D. (1965). Acta Anaesthesiologica Scandinavica, 9, 145 La Cour, D. (1966). Acta Anaesthesiologica Scandinavica, 24, 35. Williams, J. G. (1973). Stress Analysis of Polymers. London, Longman. 